\title{
An Optimized and Validated Method for Isolation and Characterization of Lymphocytes from HIV+ Human Gut Biopsies
}

\author{
Martin Trapecar, ${ }^{1, *}$ Shahzada Khan,, ${ }^{1, *}$ Nadia R. Roan, ${ }^{1,2}$ Tsui-Hua Chen, ${ }^{3}$ Sushama Telwatte, \\ Monika Deswal,, Montha Pao,, Ma Somsouk, Steven G. Deeks, \\ Peter W. Hunt, Steven Yukl, and Shomyseh Sanjabi ${ }^{1,8}$
}

\begin{abstract}
The gastrointestinal (GI) tract harbors most of the body's immune cells and is also a major HIV reservoir in ARTtreated patients. To achieve a cure, most HIV-infected cells must be identified and eliminated. While obtaining gut biopsies is a relatively noninvasive method of sampling relevant tissue for monitoring HIV activity, immune cell isolation from these limited tissue samples has proven to be challenging. Enzymatic tissue digestion is required for maximal immune cell isolation from gut biopsies. However, these enzymatic digestions can also be detrimental for preservation of cellular surface markers that are required for accurate identification of various subsets of leukocytes. In this study, we describe an optimized protocol for isolation of lymphocytes from human gut biopsies. We also discuss our validation results, which show that compared with several other collagenase preparations, the use of CSLPA maintains high lymphocyte recovery while preserving the integrity of most cellular surface antigens that we tested. Importantly, chemokine receptors that are used to characterize various subsets of T cells, which are notorious for being digested during a typical enzymatic tissue digestion, are highly preserved using this protocol.
\end{abstract}

Keywords: human gut biopsies, lymphocyte isolation, collagenase, surface antigens, CyTOF, CXCR5

\section{Introduction}

$\mathbf{M}$ ORE THAN $90 \%$ of human lymphocytes reside in the gut. ${ }^{1}$ HIV targets the gastrointestinal (GI) tract early during infection, and gut tissue significantly contributes to viral replication and persistence (see review by Khan et al. in this issue). Gut lymphocytes have unique phenotypic, functional, and survival characteristics compared with lymphocytes in other parts of the body. These properties can only be fully appreciated if phenotypic surface markers can be preserved to allow accurate characterization and comparison of the same cells isolated from different tissues.

The gut mucosa is composed of two individual compartments, the epithelium and lamina propria, and is surrounded by circular and longitudinal muscle forming the muscularis layer. The epithelial layer harbors intraepithelial lymphocytes (IELs), with very few B cells and antigen-presenting cells, whereas the underlying lamina propria harbors a vast variety of immune cells scattered throughout this connective tissue or aggregated within the lymphoid follicles. ${ }^{1}$ Most protocols for cell isolation from the intestine follow the same two distinct steps, including epithelial removal (also known as epithelial stripping) followed by enzymatic and mechanical disruption of the lamina propria. ${ }^{2-6}$ During isolation of lymphocytes from complex mucosal tissue, such as the intestine, several key factors must be considered: (1) cell yield, (2) cell viability, and (3) preservation of surface epitopes used for cellular identification through antibody staining.

To achieve maximal cell recovery, the epithelial layer must be separated from the lamina propria before further cell

\footnotetext{
${ }^{1}$ Gladstone Institute of Virology and Immunology, Gladstone Institutes, San Francisco, California.

${ }^{2}$ Department of Urology, University of California, San Francisco, San Francisco, California.

${ }^{3}$ San Francisco VA Health Care System and University of California, San Francisco (UCSF), San Francisco, California.

${ }^{4}$ Positive Health Program, Department of Medicine, University of California, San Francisco, San Francisco, California.

${ }^{5}$ Division of HIV, Infectious Diseases, and Global Medicine, Department of Medicine, University of California, San Francisco, San Francisco, California.

${ }^{6}$ Division of Gastroenterology, Department of Medicine, University of California, San Francisco, San Francisco, California.

${ }^{7}$ Division of Experimental Medicine, University of California, San Francisco, San Francisco, California.

${ }^{8}$ Department of Microbiology and Immunology, University of California, San Francisco, San Francisco, California.

*These authors contributed equally to this work.
} 
isolation. ${ }^{2,7-11}$ The epithelial layer can be easily separated from the lamina propria by the use of the reducing agent DTT and the chelating agent EDTA. However, the disruption of the remaining lamina propria and muscularis layer requires enzymatic digestion of the tissue. Enzymatic digestion with collagenase yields almost twice as many lymphocytes as mechanical disruption alone. ${ }^{8,12}$ Several types of collagenase can be used for tissue digestion, such as highly purified forms that are commercially available (e.g., the chromatographically purified CLSPA or enzyme blends known as Liberase), as well as cruder preparations such as Type I, II, and IValthough justification for the use of one type of enzyme over the others is not always provided. ${ }^{13}$

The viability and functionality of isolated cells can be profoundly affected by the proteases and prostaglandins that are released during the digestion of the mucosa. ${ }^{14}$ Therefore, in general, the viability of cells isolated from processed tissue is lower compared with cells isolated from blood or lymph nodes. Nonetheless, viability of up to $80 \%$ can be achieved when optimal conditions are used. Ficoll density gradient centrifugation can also be used to remove dead cells and debris from the final cell suspension; however, this can result in a considerable loss of cells (up to 50\%), and this procedure also prolongs the isolation time. . $^{8,13}$

Enzymatic digestion can affect the expression of surface antigens to variable degrees. ${ }^{10}$ Multiple studies have shown that collagenase II decreases the expression of CD27, ${ }^{12,15}$ whereas collagenase I decreases the expression of CD4, CD8, CD25, L-selectin, $\alpha \mathrm{E} \beta 7$, and $\alpha 4 \beta 7 .{ }^{16,17}$ Less pure preparations of collagenase often contain proteases, including trypsin and clostripain, which may also degrade cell surface proteins. ${ }^{16}$ Nevertheless, more purified forms of collagenase, such as Liberase TL, have also been documented to reduce expression of surface markers such as CD4. ${ }^{12}$ Moreover, impurities account for most of the lot-to-lot variations that require frequent revalidation of the cell isolation protocol. ${ }^{13}$

Due to the limited availability of human gut biopsy samples from HIV+ donors, as well as uncertainties in the effects of reported collagenase protocols, we evaluated the effect of EDTA and DTT, as well as various types of collagenase on the expression of surface markers present on $\mathrm{T}$ cells. We used peripheral blood mononuclear cells (PBMCs) and tonsillar lymphocytes for these optimizations, as neither require enzymatic digestion for cellular extraction, and thus undigested samples could be compared side by side with digested samples. ${ }^{16}$ Furthermore, using mass cytometry, we compared the expression level of 36 surface antigens on $\mathrm{T}$ cells after digesting tonsil cells with two different collagenase enzymes. Based on these findings, we chose the two most optimal enzymes and validated our results on human gut biopsies. In this study, we report our findings and describe the detailed protocol for the isolation of lymphocytes from human gut biopsies.

\section{Methods}

\section{Ethics statement}

Rectosigmoid biopsy samples were obtained from the SCOPE cohort at the University of California, San Francisco (UCSF). The SCOPE cohort is an ongoing longitudinal study of over 1,500 HIV-infected and uninfected adults followed for research purposes. The UCSF Committee on Human Research reviewed and approved the rectal biopsy sampling protocol for the SCOPE study (Institutional Review Board [IRB] \#10-01218, \#10-00263, or \#11-07551), and all participants provided written informed consent.

\section{Obtaining human intestinal biopsies}

Biopsy samples were retrieved from the SCOPE cohort at UCSF as previously described. ${ }^{18}$ In brief, before sigmoidoscopy and GI biopsy, participants self-administer two Fleet enemas on the morning of the procedure. Upon arriving to the clinic, study participants undergo a blood draw. An unsedated sigmoidoscopy procedure is then performed and advanced to $\sim 25 \mathrm{~cm}$ from the anus using the Olympus EndoJaw Disposable Biopsy Forceps, channel size $3.7 \mathrm{~mm}$. Biopsies are immediately placed in RPMI-1640 media (Gibco) on ice until processing.

\section{Isolation of PBMCs from human blood}

PBMCs, from the same HIV (+) study participants, were recovered by Ficoll density gradient centrifugation from fresh venous blood as described previously. ${ }^{19}$ Whole blood was spun at $1,000 \mathrm{~g}$ at RT for $10 \mathrm{~min}$ with no brake and plasma was removed. The remaining cells were diluted to 30$35 \mathrm{ml}$ with PBS, mixed, layered on $15 \mathrm{ml}$ of Ficoll, and spun at $160 \mathrm{~g}$ at RT for $20 \mathrm{~min}$ with no brake. The top $15-20 \mathrm{ml}$ of supernatant was aspirated to remove excess platelets, and the cells were spun again at $350 \mathrm{~g}$ at RT for $20 \mathrm{~min}$ with no brake. The PBMC layer was transferred to a new tube, diluted to $50 \mathrm{ml}$ with PBS, spun at $400 \mathrm{~g}$ for $8 \mathrm{~min}$ at RT, washed twice more with $50 \mathrm{ml} \mathrm{PBS}$, and counted.

\section{Isolation of cells from human tonsils}

Anonymized HIV (-) human tonsils were obtained from Cooperative Human Tissue Network (CHTN) (IRB\# 1208429) and were processed into human lymphoid aggregate cultures as described previously. ${ }^{20}$ Briefly, tonsils were minced with a plunger, passed through a $40 \mu \mathrm{m}$ strainer, and cultured in Tonsil Media, consisting of RPMI supplemented with $15 \%$ heat-inactivated fetal bovine serum (FBS), $100 \mu \mathrm{g} /$ $\mathrm{ml}$ gentamicin, $200 \mu \mathrm{g} / \mathrm{ml}$ ampicillin, $1 \mathrm{mM}$ sodium pyruvate, $1 \%$ nonessential amino acids (Mediatech), $1 \%$ GlutaMAX (Thermo Fisher), and 1\% Fungizone (Invitrogen).

\section{Protocol for the isolation of lymphocytes from human gut biopsies}

A schematic diagram of the procedure is shown in Figure 1.

\section{Equipment}

- 50-ml centrifugation tubes (Thermo Scientific/Nunc; \#339650)

- GentleMACS ${ }^{\text {Tм }}$ C tubes (Miltenyi Biotec; \#130-093237)

- Falcon ${ }^{\circledR} 5$-ml round bottom tubes with $40 \mu \mathrm{m}$ cell strainer snap cap (Corning; \#352235)

- Falcon ${ }^{\circledR}$ cell strainers; mesh size $70 \mu \mathrm{m}$ (Corning; \#352350)

- Thermal incubator with rotation unit/Incubating shaker

- GentleMACS ${ }^{\mathrm{TM}}$ Dissociator (see Notes for isolation without a dissociator) (Miltenyi Biotec; \#130-093-235)

- Vortexer

- Centrifuge

- Pipette filler

- 25-ml serological pipettes (FisherBrand; \#13-676-10 M)

- 5-ml syringes with Luer Lock Tip (BD; \#BD 301029) 


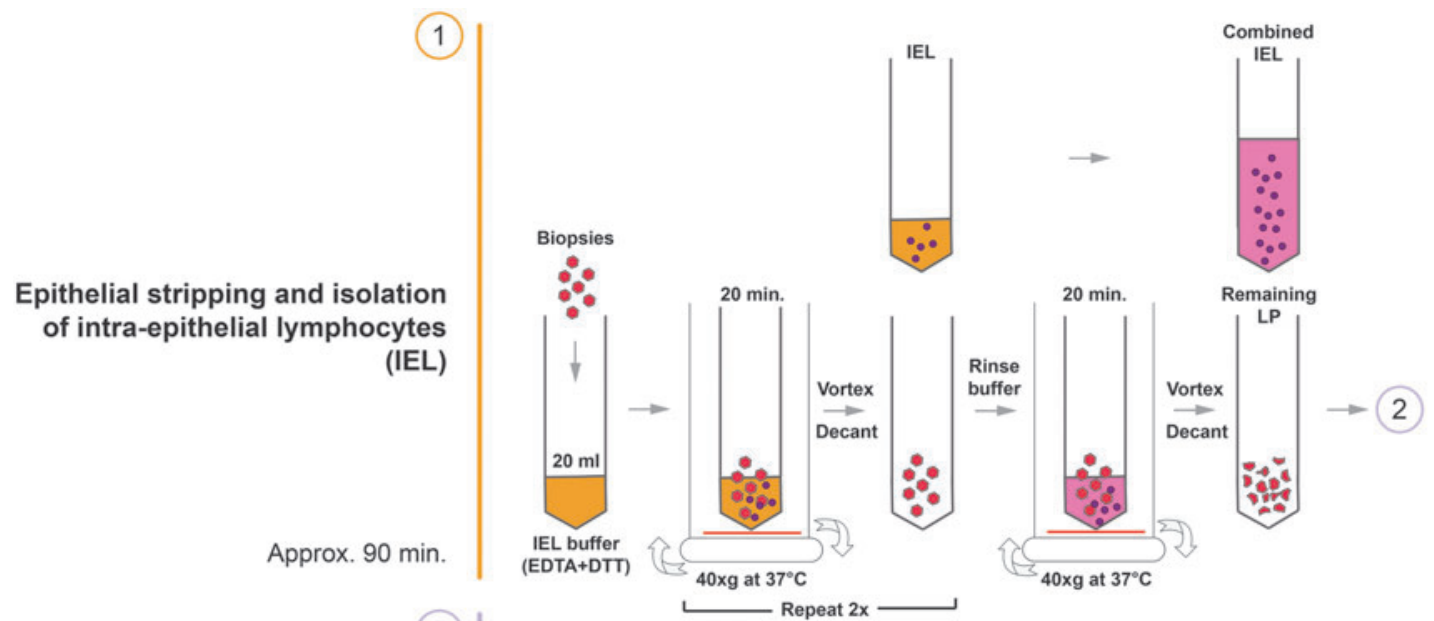

Enzymatic digestion of the lamina propria

Approx. $50 \mathrm{~min}$.

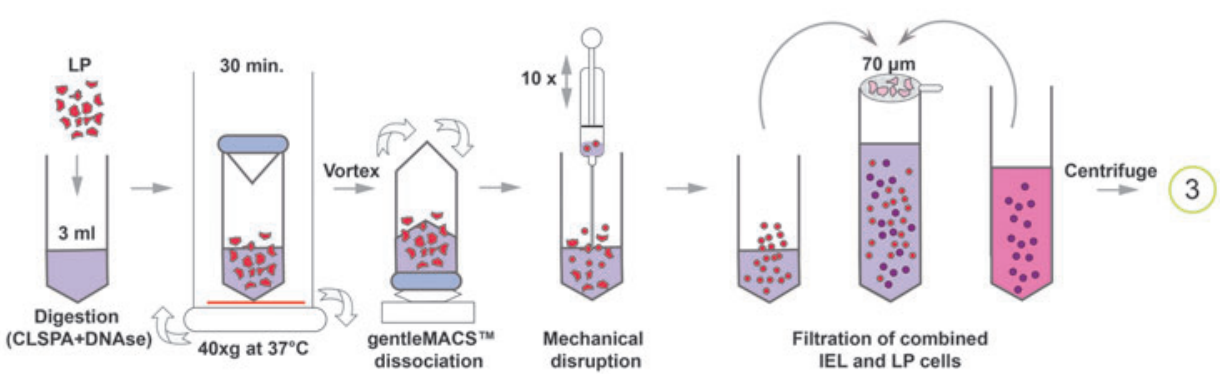

DNAse treatment
and final filtration

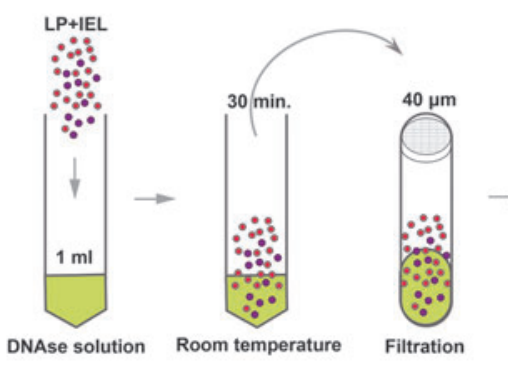

FIG. 1. Lymphocyte isolation scheme.

- 20G blunt needles (BD; \#BD 305180)

- Cell scraper (Thermo Scientific/Nunc; \#179693)

- Forceps

Reagents

(a) Predigesting solution for epithelial stripping and isolation of IEL/(40 $\mathrm{ml}$ per sample)-referred to as IEL buffer

(i) sterile DPBS, $\mathrm{Ca}^{2+}$ and $\mathrm{Mg}^{2+}$ free (Corning; \#21040-CV)

(ii) DTT $10 \mathrm{mM}$ (Sigma-Aldrich; \#3483-12-3)

(iii) EDTA $5 \mathrm{mM}$ (Sigma-Aldrich; \#60-00-4)

(iv) HEPES $10 \mathrm{mM}$ (Fisher BioReagents; \#7365-45-9)

(v) FBS (5\%) (GE Healthcare Life Sciences SH30070.01)

(b) Rinse buffer (20 $\mathrm{ml}$ per sample + additional buffer for washes)

(i) RPMI-1640 (Corning; \#10-040-CMR)

(ii) HEPES $10 \mathrm{mM}$ (Fisher BioReagents; \#7365-45-9)

(iii) FBS (5\%) (GE Healthcare Life Sciences SH30070.01)

(c) Digestion solution $(3 \mathrm{ml}$ per sample each round of digestion-normally two rounds are needed) (i) RPMI-1640 (Corning; \#10-040-CMR)

(ii) HEPES $10 \mathrm{mM}$ (Fisher BioReagents; \#7365-45-9)

(iii) CLSPA collagenase $(0.5 \mathrm{mg} / \mathrm{ml})$ (Worthington; \#LS005273)

(iv) DNAse $(7.5 \mu \mathrm{g} / \mathrm{ml})$ (Roche; \#10104159001)

(d) DNAse solution (1 $\mathrm{ml}$ per sample)

(i) RPMI-1640 (Corning; \#10-040-CMR)

(ii) HEPES $10 \mathrm{mM}$ (Fisher BioReagents; \#7365-45-9)

(iii) DNAse $(100 \mu \mathrm{g} / \mathrm{ml})$ (Roche; \#10104159001)

(e) Fluorescence-activated cell sorting (FACS) buffer: $1 \%$ FBS in $1 \times$ PBS

\section{Procedure}

(a) Epithelial stripping and isolation of IELs

(1) Transfer the biopsies or whole tissue pieces into a $50-\mathrm{ml}$ conical tube containing $20 \mathrm{~mL}$ of IEL buffer, prewarmed to $37^{\circ} \mathrm{C}$.

(2) Incubate the sample horizontally for $20 \mathrm{~min}$ at $37^{\circ} \mathrm{C}$ under continuous rotation $(40 \mathrm{~g})$ using an incubating shaker.

(3) Mix well for $10 \mathrm{~s}$ using a vortexer. 
(4) Use a 25-ml serological pipette to aspirate the biopsies and tissue pieces with buffer and let the pieces settle to the tip of the pipette. Return the pieces to the tube while transferring the IEL buffer supernatant, which contains the stripped epithelium and IELs, into a new 50-ml conical tube placed on ice.

(5) Add $20 \mathrm{ml}$ of fresh prewarmed IEL buffer to the biopsies and incubate the sample for another $20 \mathrm{~min}$ at $37^{\circ} \mathrm{C}$ under continuous rotation $(40 \mathrm{~g})$ using an incubating shaker.

(6) During the incubation period, spin down the 50$\mathrm{ml}$ tube that contains the collected IEL supernatant at $300 \mathrm{~g}$ for $5 \mathrm{~min}$. Dislodge the pellet, add $5 \mathrm{ml}$ of rinse buffer to the isolated cells and place the tube back on ice.

(7) After the second round of the 20-min incubation of the biopsies is completed, mix the tube well for $10 \mathrm{~s}$ using a vortexer and repeat step number 4 . If you have already done so, continue with step 8 .

(8) Add $20 \mathrm{ml}$ of prewarmed rinse buffer to the biopsies and incubate the samples for a third time for $20 \mathrm{~min}$ at $37^{\circ} \mathrm{C}$ under continuous rotation $(40$ $g$ ) using an incubating shaker.

(9) During the incubation, repeat step 6 with the supernatants of the 2nd incubation.

(10) When the 3rd round of incubation is completed, repeat step 3 and 4 , only this time transfer the remaining pieces of tissue into a gentleMACS ${ }^{\mathrm{TM}}$ $\mathrm{C}$ tube and proceed with the protocol for isolating cells from the lamina propria.

(11) Repeat step 6 with the supernatants of the final incubation round and then combine all the isolated IEL cells into one 50-ml tube.

(12) Pass the cell suspension through a $70 \mu \mathrm{m}$ cell strainer, placed on a new 50-ml conical tube. Wash the cell strainer with $20 \mathrm{ml}$ of rinse buffer.

(13) Centrifuge the suspension at $300 \mathrm{~g}$ for $5 \mathrm{~min}$, discard the supernatant, and resuspend the pellet in the desired volume and buffer for further application or for combining them with the cells isolated from the lamina propria.

(b) Isolation of cell populations from the lamina propria

(1) Add $3 \mathrm{ml}$ of the digestion solution, prewarmed to $37^{\circ} \mathrm{C}$, to the gentleMACS ${ }^{\mathrm{TM}} \mathrm{C}$ tube that contains the remaining pieces, and incubate sample for $30 \mathrm{~min}$ at $37^{\circ} \mathrm{C}$ under continuous rotation $(40 \mathrm{~g})$ using an incubating shaker.

(2) Mix well for $10 \mathrm{~s}$ using a vortexer

(3) Insert the $\mathrm{C}$ tube with the digested pieces into the gentleMACS $^{\mathrm{TM}}$ Dissociator and run program "m_intestine_01".

(4) Spin the tube briefly to prevent cell accumulation near the cap.

(5) Using a 5-ml syringe and a blunt 20G needle, aspirate the sample and gently return it to the tube. Repeat this step of mechanical dissociation 10 times.

(6) Add $5 \mathrm{ml}$ of rinse buffer to the gentleMACS ${ }^{\mathrm{TM}} \mathrm{C}$ tube and pass the cell suspension through a $70 \mu \mathrm{m}$ cell strainer, placed on a new $50-\mathrm{ml}$ conical tube. Wash the gentleMACS ${ }^{\mathrm{TM}} \mathrm{C}$ tube with $10 \mathrm{ml}$ of rinse buffer and pass it through the strainer.
(7) Take a sterile cell scraper and gently scrape the surface of the strainer and inspect it for remaining pieces of tissue. If visible tissue remains, transfer the collected tissues with forceps back into the gentleMACS ${ }^{\mathrm{TM}} \mathrm{C}$ tube and repeat steps $1-8$ of the lamina propria isolation protocol.

(8) Centrifuge the 50-ml tube containing the digested lamina propria at $300 \mathrm{~g}$ for $5 \mathrm{~min}$. After centrifugation, discard the supernatant and resuspend the pellet of isolated cells in $5 \mathrm{ml}$ of rinse buffer.

(9) Combine all the collected lamina propria cells and proceed with the next step.

(c) Incubation with DNAse

(1) Centrifuge the combined IEL and LP cells (or centrifuge separately if IEL and LP cells need to be analyzed individually) at $300 \mathrm{~g}$ for $5 \mathrm{~min}$. Discard the supernatant and resuspend all the isolated cells in $1 \mathrm{ml}$ of the DNAse solution.

(2) Incubate at room temperature (RT) for $30 \mathrm{~min}$.

(3) Centrifuge cells at $300 \mathrm{~g}$ for $5 \mathrm{~min}$, discard the supernatants, and resuspend in $3 \mathrm{ml}$ of FACS buffer.

(4) Pass the cells through the $40 \mu \mathrm{m}$ cell strainer snap cap placed on the 5-ml round bottom tubes. Wash the strainer with $2 \mathrm{ml}$ of FACS buffer.

(5) Centrifuge at $300 \mathrm{~g}$ for $5 \mathrm{~min}$, discard the supernatant and resuspend the cells in the desired medium for further application.

Notes

- All solutions, except the FACS buffer and DNAse solution, need to be prewarmed to $37^{\circ} \mathrm{C}$

- Thorough vortexing is needed for successful disruption of the epithelial layer during epithelial stripping and isolation of IEL.

- Due to the adherent and clumping nature of mucosal samples, many cells get trapped during cell straining. Therefore, thorough rinsing of the cell strainers is important for optimal cell recovery.

- For enhanced cell recovery, it is advisable to collect separately all the discarded supernatants throughout the isolation procedure. At the end of the isolation, these can be filtered, centrifuged, and combined with the remaining cells.

- If a gentleMACS ${ }^{\mathrm{TM}}$ Dissociator is not available, biopsies can be transferred into a 50-ml conical tube after epithelial stripping, incubated with the digestion solution for $30 \mathrm{~min}$, and then vortexed for $30 \mathrm{~s}$.

- Excessive foaming should be avoided at all stages.

- Including serum in the digestion solution decreases enzyme activity.

- The final round of incubation with DNAse, followed by $40 \mu \mathrm{m}$ filtration, greatly improves viscosity and reduces the need for Ficoll gradient centrifugation

\section{Characterization of surface marker expression with multicolor flow cytometry}

Single cell suspensions were washed twice with PBS, followed by staining with the LIVE/DEAD ${ }^{\mathrm{TM}}$ Fixable Aqua Dead Cell Stain Kit (405 nm excitation; Invitrogen) for 15 min at RT. Stained cells were washed twice with PBS, blocked for $\mathrm{Fc}$ receptors (Human BD Fc Block ${ }^{\mathrm{TM}}$; BD 
Biosciences) for $10 \mathrm{~min}$ at RT, and stained with a mixture of fluorochrome-conjugated anti-human primary Abs: BV650 CD4 (SK3), BV605 CD8 (SK1), BV421 CD103 (Ber-ACT8), APC-H7 CD45RA (HI100), AF647 CXCR5 (RF8B2), PECy7 PD1 (EH12.1) or PE-Cy7 CXCR3 (1C6/CXCR3), Percp-Cy5.5 CD3 (UCHT1), PE CCR6 (11A9), and FITC CD69 (FN50) from BD Biosciences. Stained cells were washed once and flow cytometry was performed with an LSR II flow cytometer and FACSDiva software (BD). Acquired data were analyzed using FlowJo software (TreeStar).

\section{Characterization of surface marker expression with mass cytometry}

Aliquots of tonsil cells from the same donor were processed either with a protocol previously optimized for fibroblast/endometrial gland isolation using collagenase type $\mathrm{I}^{21}$ or with the protocol using CLSPA as described in this study. Digested tonsil cells were then analyzed by mass cytometry (CyTOF), as previously described. ${ }^{22}$ For staining, a total of 38 Abs were used in the panel, 33 of which have been validated in a recent report. $^{22}$ Three additional Abs (LAG3, CD103, and TIGIT) were further validated and used with the Ab panel in this study.

\section{Results}

\section{Enzyme selection}

Various types of collagenase enzymes have been used to isolate immune cells from mouse $\mathrm{e}^{13,23}$ and human intestinal tissue $^{2,16}$ with varying degrees of cell surface marker preservation. We sought to determine which enzyme would best preserve surface marker expression on human $\mathrm{T}$ cells. For this comparison, we used four different types of collagenase enzymes: type I, II-s, IV, and CLSPA. Human tonsil cells $\left(\sim 5.0 \times 10^{6}\right)$ were incubated with each of the enzymes in digestion solution (RPMI-1640 with HEPES and DNAse, see methods section) for $30 \mathrm{~min}$ at $37^{\circ} \mathrm{C}$, followed by washing and staining for flow cytometric analyses. We found that CXCR5, a chemokine receptor used for identifying $\mathrm{T}$ follicular helper (Tfh) cells, ${ }^{24}$ was highly susceptible to collagenase digestion (Fig. 2A). Collagenase I, II-s, and IV almost completely digested away CXCR5, whereas digestion with CLSPA preserved this receptor on CD4 T cells. CLSPAdigested cells had high, intermediate, and low CXCR5expressing populations among CD4 T cells, and the pattern was similar to that found on cells that were not treated with any enzyme (Fig. 2A). As EDTA and DTT are used for stripping the epithelial layer from gut samples, ${ }^{2,7,8}$ we next tested whether the combination of this treatment would affect CXCR5 surface expression. This stripping step helps to reduce the amount of time the LP has to be digested with collagenase. As our gut protocol combines the isolated IELs with those isolated from LP (Fig. 1), it should recover lymphocytes from all portions of the gut biopsy. We found no significant effect of this stripping step on the levels of CXCR5 expression (Fig. 2B). Similar results were obtained from three different tonsil donor samples, which excluded any donor-specific effect observed after enzymatic treatments.

Identification of various subsets of immune cells relies on successful detection of different surface markers. To determine whether cells isolated after CLSPA digestion also retain other cell surface markers used to characterize mucosal T cells, we used a mass cytometry panel and characterized the expression of 38 different cell surface markers found on various subsets of CD4 T cells. ${ }^{22}$ Compared with collagenase type I, CLSPA had less impact on almost all of the cell surface markers (Fig. 2C). Most importantly, Type I collagenase highly reduced the expression of CD4, which was essentially unchanged with CLSPA digestion. Other cell surface markers for identifying the major subsets of CD4 $\mathrm{T}$ cells, such as CD45RO, CD62L, and HLA-DR, were also best preserved with CLSPA digestion (Fig. 2C).

\section{Validation of CLSPA as the preferred collagenase for lymphocyte isolation from rectosigmoid biopsies}

We used human rectosigmoid biopsies from HIV+ participants to further validate CLSPA as a preferred enzyme for isolation and characterization of T cells from the gut. Similar to the tonsil cells, $\mathrm{T}$ cells isolated from gut biopsies using CLSPA digestion retained expression of CXCR5, whereas Type IV collagenase completely disrupted CXCR5 surface expression on gut $\mathrm{T}$ cells from the same individual (Fig. 3A). $\mathrm{CD}^{+}$cells isolated from CLSPA or Type IV-digested gut biopsies had similar and clearly detectable expression of many of the other cell surface markers, including CD3, CD4, CD8 (not shown), CD45RA, CD103, CD69, and PD1. Consistent with the CyTOF data, expression of CD4, CCR6, CD103, and PD1 was better preserved in CLSPA-digested compared with Type IV-digested biopsies. For unknown reasons, CXCR3 expression was slightly enhanced in CD4 T cells isolated after collagenase digestion, although this level was similar in $\mathrm{CXCR}^{+} \mathrm{CD} 4 \mathrm{~T}$ cells isolated with either of the enzymes (Fig. 3B).

\section{Cell recovery and viability}

We collected 18-26 rectosigmoid biopsies from each individual. All the biopsies were utilized for obtaining a single cell suspension after CLSPA digestion. Processed and digested gut samples retained about $70 \%$ viability, and expression of cell surface markers were comparable to those found on PBMCs from the same participant that was stained with the same cocktail of antibodies (Fig. 4A). On average, we obtained $\sim 1.5 \times 10^{6}$ total cells per biopsy (Fig. 4B), with a range of $60 \%-85 \%$ cell viability (Fig. 4C). Among total live cells, $4 \%-17 \%$ were $\mathrm{CD}^{+}$cells (Fig. 4D), of which $\sim 40 \%$ were also $\mathrm{CD}^{+}$(Fig. 4A). Therefore, based on these numbers, $\sim 20,000$ to 30,000 live $\mathrm{CD} 4 \mathrm{~T}$ cells are recoverable from a total of $1.5 \times 10^{6}$ cells that can be obtained from each rectosigmoid jumbo forceps biopsy from HIV+ donors.

\section{Discussion}

Cell isolation from human tissue, which relies on the ability to digest tissue while preserving various cellular surface antigens, is central for research done on tissues, such as investigating different cellular reservoirs for HIV in the gut. Comparing results from immunohistochemistry and flow cytometry, it is estimated that only $20 \%$ of T cells can be recovered from human gut biopsies by enzymatic digestion. ${ }^{25}$ In this study, we report an improved protocol for the isolation of lymphocytes from human gut biopsies and describe the 
A

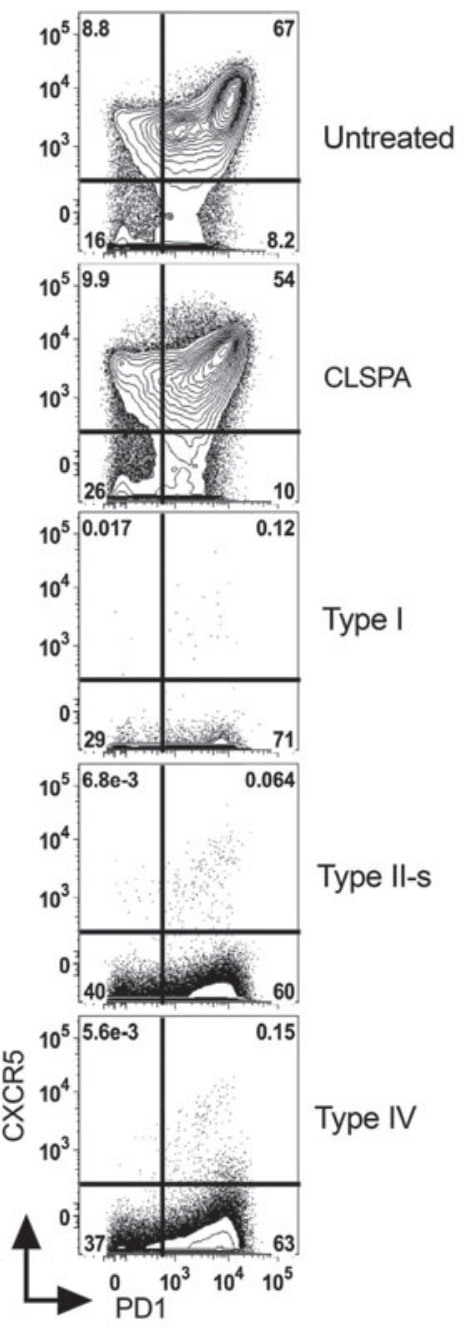

B

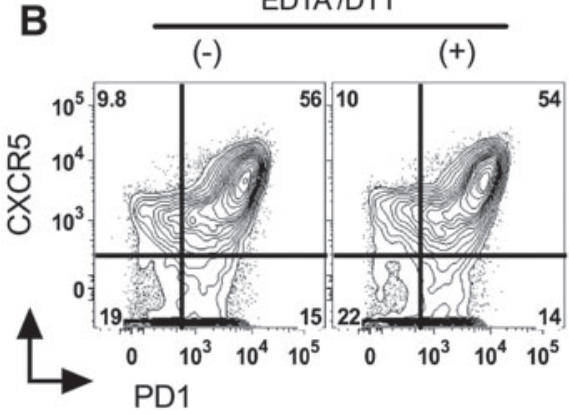

C
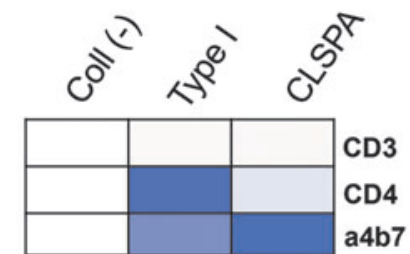

a4b7

CCR2

CCR4

CCR5

CCR6

CCR7

CCR9

CD103

CD127

CD25

CD27

CD28

CD29

CD38

CD45RO

CD45RA

CD49d

CD57

CD62L

CD69

CD7

CXCR3

CXCR4

CXCR5

HLA-DR

ICOS

LAG3

OX040

PD1

TIGIT

Fold over untreated

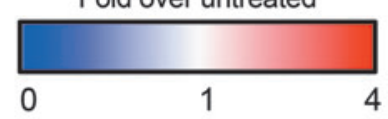

FIG. 2. Effect of various collagenase enzymes on CD4 $\mathrm{T}$ cell surface antigens. (A) Human tonsil cells collected from the same donor were digested for $30 \mathrm{~min}$ at $37^{\circ} \mathrm{C}$ with $500 \mathrm{U} / \mathrm{ml}$ of either collagenase type I, II-s, IV, or CLSPA. Representative flow plots from one of four different donors with similar results are shown. (B) Tonsil cells were first incubated at $37^{\circ} \mathrm{C}$ for $20 \mathrm{~min}$ with stripping buffer containing EDTA and DTT, or were left on ice in FACS buffer without EDTA and DTT. Cells from both conditions were incubated in digestion media containing CLSPA for $30 \mathrm{~min}$, followed by analysis using flow cytometry. (C) Heat map showing fold change in MFIs of treated over untreated CD4 $\mathrm{T}$ cells. Tonsil cells were treated with either collagenase type I or CLSPA as in A, or were left untreated. Surface antigens were analyzed by mass cytometry. All cells were first gated on live cells and singlets, followed by gating on $\mathrm{CD}^{+} \mathrm{CD}^{+}$cells. Blue indicates reduction and red indicates enhancement in levels of indicated surface molecules, as compared with untreated cells. The experiments described in each panel in this figure used tonsils from different donors. FACS, fluorescence-activated cell sorting; MFI, median fluorescence intensity effect of various types of collagenase on the expression of $\mathrm{T}$ cell surface markers. We tested four different collagenases and have identified CLSPA, which is a highly purified bacterial collagenase from Clostridium histolyticum, as the collagenase that causes the least damage to cell surface antigens. A two-step digestion protocol, in which the epithelium is first stripped for IEL isolation, followed by digestion of LP with CLSPA, maintains a high yield of live cells, estimated to be around $1.0-2.5 \times 10^{6}$ cells per biopsy. Our results indicate that EDTA and DTT do not alter the expression of CXCR5 and PD1, whereas others have shown that DTT can decrease the expression of CD11a and CD49d. ${ }^{16}$ Therefore, the effect of EDTA and DTT should be further verified for each specific surface antigen of interest.

To achieve increased enzyme activity, we omitted the use of serum during the enzyme digestion phase ${ }^{10}$ and used preheated $\left(37^{\circ} \mathrm{C}\right)$ solutions at all times. In accordance with other reports, we confirm (data not shown) that one of the most important factors in achieving high cell yields is the temperature of the digestion solution. ${ }^{13}$ Moreover, we 
FIG. 3. Improved retention of surface antigens on cells isolated from CLSPAdigested human rectosigmoid biopsies. (A) Flow cytometry plots of CD4 $\mathrm{T}$ cells from $\mathrm{PBMC}$ or rectosigmoid biopsies collected from an HIV (+) participant (PID \#2). Tonsil cells from an HIV $(-)$ donor are also shown. The same number of rectosigmoid biopsies were digested with either collagenase IV or CLSPA. (B) Detection of various surface markers on $\mathrm{CD}^{+}$cells in rectosigmoid biopsies from (A). $\mathrm{CD}^{+}$cells from PBMC are also shown as an undigested control. Representative plots of CLSPA digestion of biopsies from one of six different HIV (+) participants are shown. PBMC, peripheral blood mononuclear cell.
FIG. 4. Cell recovery from CLSPA-digested rectosigmoid biopsies. (A) Gating strategy for isolating $\mathrm{CD}^{+}$cells from PBMC and rectosigmoid biopsies of HIV $(+)$ participants. (B) Total cells recovered per biopsy. At least 18 biopsies were used to generate total cell count per participant, and then the cell number was divided by the number of biopsies from each participant. (C) Percentages of live (Aquamine -), and (D) $\mathrm{CD}^{+}$in total cells obtained from CLSPA-digested rectosigmoid biopsies from each participant.
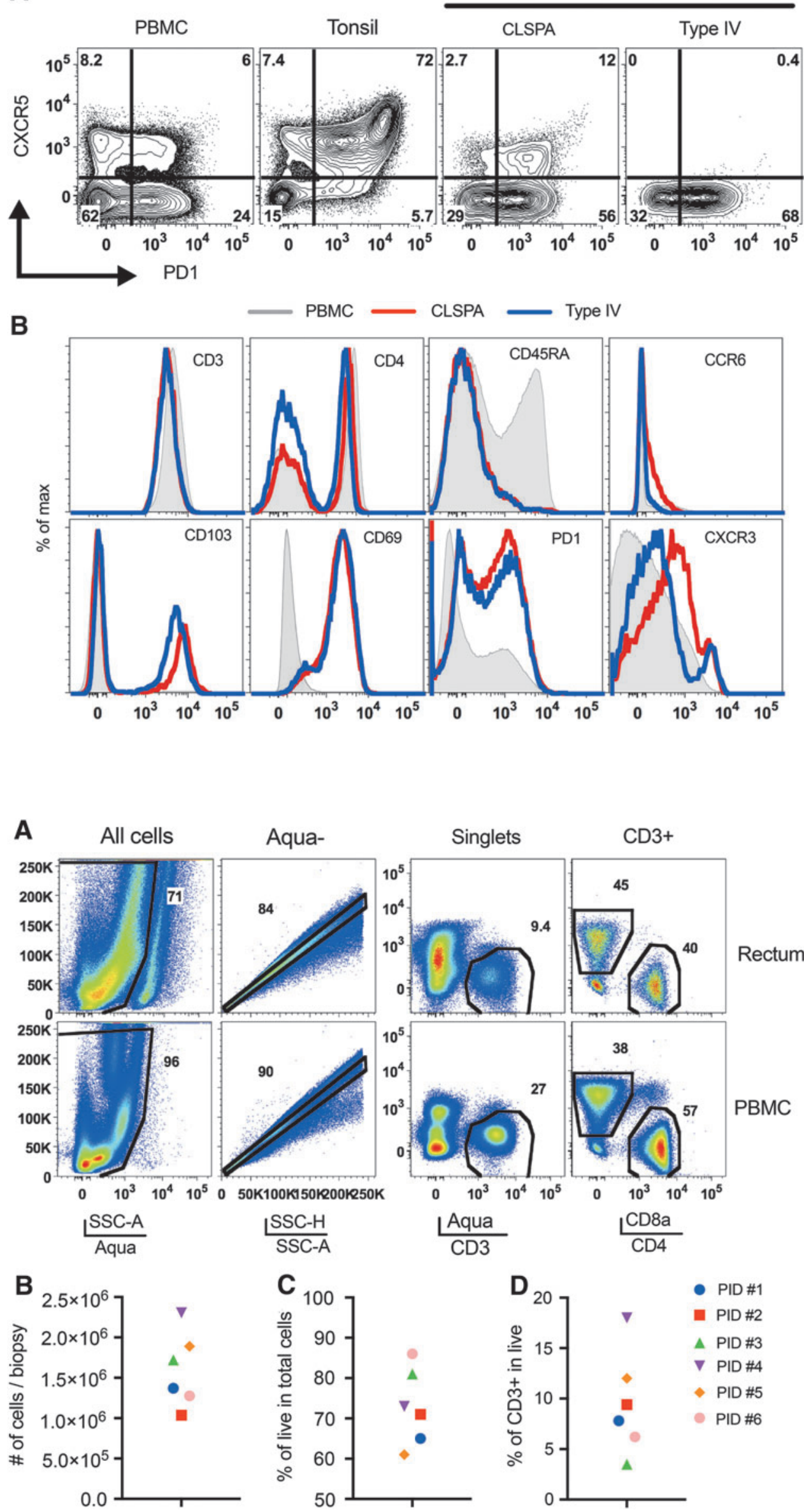
were able to greatly reduce the viscosity of the cell suspension by introducing an additional round of incubation with DNAse alone, followed by $40 \mu \mathrm{m}$ filtration. This filtration step was especially useful to facilitate downstream applications involving cell sorting and flow cytometry. In addition, this step eliminated the need for gradient centrifugation and may have contributed to better recovery of total cells and thus higher number of live $\mathrm{T}$ cells available for cell sorting.

Our optimized protocol did not assess the actual functionality of recovered cells and how this may have been affected by the enzymatic digestion that is required for cell isolation. Although multiple studies have used enzymatic digestion to isolate apparently functional cells from tissues (including gut lymphocytes), conflicting evidence exists with regard to the effect of cell isolation technique on the functionality of the cells. ${ }^{14} \mathrm{We}$ found that collagenase digestion increased the surface expression of a few proteins, suggesting that the enzymatic digestion protocol may alter expression, stability, and/or trafficking of some cellular proteins. It has been noted that levels of HIV RNA and HIV RNA per provirus (normalized to GAPDH), but not HIV DNA were higher in collagenase-digested total gut cells than in intact biopsies, suggesting that collagenase digestion may also affect transcription of HIV or housekeeping genes. ${ }^{26}$ Therefore, isolation of immune cells for gene expression or functional assessment requires careful optimization and controls. At the same time, it can be difficult to perform and interpret these controls, since studies performed on cells that naturally exist in suspension-such as PBMCsmay not recapitulate what happens when adherent cells are released from tissues. Moreover, some studies cannot be performed without tissue dissociation and/or cell isolation, and it is unclear whether there are suitable alternatives to enzymatic digestion-such as those that utilize only mechanical dissociation-or how these alternative methods affect cell recovery, viability, surface markers, or gene expression. Thus, there is a critical need for further development of isolation techniques that preserve cellular states, and an improved understanding of how cell isolation impacts gene expression and functional activity of the cells.

\section{Acknowledgments}

This work was supported by California HIV/AIDS Research Program IDEA Award (CHRP ID15-GI-059), as part of the amfAR Institute for HIV Cure Research, with funding from amfAR grant number 109301, and by the National Institutes of Health (NIH; DP2 AI112244) to SS, and (1R01DK108349-01 and U01AI034989) to SY. SK was also supported by a CFAR Mentored Scientist Award (P30 AI027763). This publication was made possible with the help from the University of California San Francisco, Gladstone Institute of Virology \& Immunology Center for AIDS Research (CFAR), an NIHfunded program (P30 AI027763), NIH S10 RR028962, and the James B. Pendelton Charitable Trust, all supporting the Gladstone Flow Core and cell sorter. The authors would also like to thank the study participants and the SCOPE study coordinators, including Rebecca Hoh, for their efforts in recruiting the participants for these studies.

\section{Author Disclosure Statement}

All authors declare no conflict of interest.

\section{References}

1. Mowat AM, Agace WW: Regional specialization within the intestinal immune system. Nat Rev Immunol 2014;14: 667-685.

2. Bull DM, Bookman MA: Isolation and functional characterization of human intestinal mucosal lymphoid cells. J Clin Invest 1977;59:966-974.

3. Reissig S, Hackenbruch C, Hovelmeyer N: Isolation of T cells from the gut. Methods Mol Biol 2014;1193:21-25.

4. Sheridan BS, Lefrancois L: Isolation of mouse lymphocytes from small intestine tissues. Curr Protoc Immunol 2012; Chapter 3:Unit 319.

5. Lefrancois L, Lycke N: Isolation of mouse small intestinal intraepithelial lymphocytes, Peyer's patch, and lamina propria cells. Curr Protoc Immunol 2001; Chapter 3:Unit 319.

6. Lundqvist C, Hammarstrom ML, Athlin L, Hammarstrom $\mathrm{S}$ : Isolation of functionally active intraepithelial lymphocytes and enterocytes from human small and large intestine. J Immunol Methods 1992;152:253-263.

7. Stern BK, Jensen WE: Active transport of glucose by suspensions of isolated rat intestinal epithelial cells. Nature 1966;209:789-790.

8. Bland PW, Richens ER, Britton DC, Lloyd JV: Isolation and purification of human large bowel mucosal lymphoid cells: Effect of separation technique on functional characteristics. Gut 1979;20:1037-1046.

9. Fiocchi C, Battisto JR, Farmer RG: Gut mucosal lymphocytes in inflammatory bowel disease: Isolation and preliminary functional characterization. Dig Dis Sci 1979;24: 705-717.

10. Couter CJ, Surana NK: Isolation and flow cytometric characterization of murine small intestinal lymphocytes. J Vis Exp 2016; DOI:10.3791/54114.

11. Mosley RL, Klein JR: A rapid method for isolating murine intestine intraepithelial lymphocytes with high yield and purity. J Immunol Methods 1992;156:19-26.

12. Schreurs R, Drewniak A, Bakx R, et al.: Quantitative comparison of human intestinal mononuclear leukocyte isolation techniques for flow cytometric analyses. J Immunol Methods 2017;445:45-52.

13. Goodyear AW, Kumar A, Dow S, Ryan EP: Optimization of murine small intestine leukocyte isolation for global immune phenotype analysis. J Immunol Methods 2014;405: 97-108.

14. Gibson PR, Hermanowicz A, Verhaar HJ, Ferguson DJ, Bernal AL, Jewell DP: Isolation of intestinal mononuclear cells: Factors released which affect lymphocyte viability and function. Gut 1985;26:60-68.

15. Shen C, Xu H, Alvarez X, Lackner AA, Veazey RS, Wang $\mathrm{X}$ : Reduced expression of CD27 by collagenase treatment: Implications for interpreting b cell data in tissues. PLoS One 2015;10:e0116667.

16. Van Damme N, Baeten D, De Vos M, et al:: Chemical agents and enzymes used for the extraction of gut lymphocytes influence flow cytometric detection of $\mathrm{T}$ cell surface markers. J Immunol Methods 2000;236:27-35.

17. Abuzakouk M, Feighery C, O'Farrelly C: Collagenase and Dispase enzymes disrupt lymphocyte surface molecules. J Immunol Methods 1996;194:211-216. 
18. Somsouk M, Estes JD, Deleage C, et al.: Gut epithelial barrier and systemic inflammation during chronic HIV infection. AIDS 2015;29:43-51.

19. Yukl SA, Shergill AK, McQuaid K, et al.: Effect of raltegravir-containing intensification on HIV burden and Tcell activation in multiple gut sites of HIV-positive adults on suppressive antiretroviral therapy. AIDS 2010;24:2451-2460.

20. Jekle A, Keppler OT, De Clercq E, Schols D, Weinstein M, Goldsmith MA: In vivo evolution of human immunodeficiency virus type 1 toward increased pathogenicity through CXCR4-mediated killing of uninfected CD4 T cells. J Virol 2003;77:5846-5854.

21. Chen JC, Johnson BA, Erikson DW, et al:: Seminal plasma induces global transcriptomic changes associated with cell migration, proliferation and viability in endometrial epithelial cells and stromal fibroblasts. Hum Reprod 2014;29: 1255-1270.

22. Cavrois M, Banerjee T, Mukherjee G, et al:: Mass cytometric analysis of HIV entry, replication, and remodeling in tissue CD4+ T cells. Cell Rep 2017;20:984-998.

23. Davies MD, Parrott DM: Preparation and purification of lymphocytes from the epithelium and lamina propria of murine small intestine. Gut 1981;22:481-488.
24. Breitfeld D, Ohl L, Kremmer E, et al.: Follicular B helper T cells express CXC chemokine receptor 5, localize to B cell follicles, and support immunoglobulin production. J Exp Med 2000;192:1545-1552.

25. Preza GC, Yang OO, Elliott J, Anton PA, Ochoa MT: T lymphocyte density and distribution in human colorectal mucosa, and inefficiency of current cell isolation protocols. PLoS One 2015;10:e0122723.

26. Yukl SA, Sinclair E, Somsouk M, et al.: A comparison of methods for measuring rectal HIV levels suggests that HIV DNA resides in cells other than CD4+ T cells, including myeloid cells. AIDS 2014;28:439-442.

Address correspondence to: Shomyseh Sanjabi

Gladstone Institute of Virology and Immunology, Gladstone Institutes 1650 Owens Street San Francisco, CA 94158

E-mail: shomyseh.sanjabi@gladstone.ucsf.edu 